\title{
Simulation of the collapse of an underwater explosion bubble under a circular plate
}

\author{
Kit-Keung Kan, James H. Stuhmiller and Philemon C. Chan* \\ Jaycor, Inc., 3394 Carmel Mountain Road, San Diego, CA 92121, USA
}

Received 21 November 2000

Revised 6 Septmeber 2002

\begin{abstract}
A two-fluid, computational fluid dynamics study of the phenomena of bubble collapse under a submersed flat plate has been performed. In order to handle the rapidly changing bubble-water interface accurately, second order upwind differencing is used in calculating the advection term. Good agreement with experimental data is obtained for the pressure distribution on the plate. The computational results provide insight into the phenomenology of the jet impact, the formation of a radial hydraulic jump, and the complex interaction of that hydraulic jump with the collapsing toroidal bubble.
\end{abstract}

\section{Introduction}

The damage of cavitation bubbles to hydraulic machinery is well known. The damage mechanism is due to the formation of a water jet that impinges on the nearby solid surface when the bubble collapses asymmetrically. Kornfeld and Suvorov [1], Snay and Christian [2], Naude and Ellis [3], Benjamin and Ellis [4], and Plesset and Chapman [5] are the pioneers in studying this jetting phenomenon. Recent studies have been directed towards more quantitative understandings of the bubble-structure interaction and the loading mechanism of the jet (Tomita and Shima [6], Blake, Taib and Doherty [7], Blake and Gibson [8], and Duncan and Zhang [9]).

A bubble generated by an underwater explosion behaves in a similar way and can be characterized by its volume and pressure at the bubble maximum, as described by Rayleigh's bubble theory [10]. Explosion bubble experiments were performed by Goertner, Thrun and Berry [11] at the Naval Surface Warfare Center (NSWC) in 1987. These experiments have provided the first detailed mapping of the pressure loading on a solid surface by the collapsing jet and are of great importance in the understanding of the bubble-structure interaction.

Numerical simulations of the bubble collapse and coupled bubble-structure interaction are still in a developing stage. Most of the past investigations rely on the potential flow approximation (Blake and Prosperetti [12], Chahine [13,14]). Such methods work well as long as the bubble is a simply connected entity. After the jet develops and touches the other side of the bubble, the bubble becomes a torus and hence a doubly connected object, and the potential approximation must be modified before continuing. While modifying the connectivity by introducing cuts in the flow field is possible (Chahine [14], Best [15]), the process will become increasingly complex and cumbersome as the bubble breaks up into smaller fragments, not to mention their effects on accuracy.

An approach based on two-fluid flow computational fluid dynamics (CFD) is attractive because it poses no restriction on the motion of the bubble surface. It can handle the jetting and the subsequent complicated fragmentation of the bubble without intervention. However, the rapid changes of the flow in space and time at the jetting surface demand highly accurate treatments in many aspects of the flow, or else, prohibitively fine grids are needed in order to

*Corresponding author. Tel.: +1 858720 4000; E-mail: philemon.chan@Titan.com. 
obtain reasonable results. Earlier attempts of two-phase flow simulation by Chan [16] have captured some qualitative features of the collapse in a grid of modest size, but yielded an incorrect development of the jet.

We report here an improvement of Chan's work [16] that leads to an overall good agreement with experimental data. This improvement is achieved by the use of a second order upwind advections and of a mass flux that generates no net mass at the interface in pure advection of the flow.

\section{Experiments}

The experiments to be studied by the present simulation effort are the series of tests carried out in the Hydrotank facility at the NSWC White Oak Laboratory in 1987 (Goertner, Thrun and Berry [11]). Placed directly below a submersed circular flat plate with controlled standoff, small explosives were detonated to generate the bubbles. The tests can be grouped into two sets, a set with the air above the free surface left in a near normal atmospheric condition and the other with the pressure reduced. Repeatability of the test was demonstrated with explosions at similar test conditions. High-speed movies of the bubble were taken and pressure loading on the plate was recorded.

\section{Computational method}

The computer code used for the simulation is JAYCOR's Equation Independent Transient Analysis Computer Code (EITACC). It is a finite difference CFD code for solving the time dependent, Reynolds averaged, compressible, Navier-Stokes equations for the flow of gas and liquid in complex three-dimensional geometry. It is organized in such a way that one can choose different basic equations at ease to model the physical phenomenon at hand. The numerical method is similar to the semi-implicit method employed by Liles and Reed [17], in which the pressure solution is fully implicit, while the velocity advection and mass flux are treated explicitly. The application of EITACC for two-phase dispersed bubbly flow has been reported earlier by Chan, Masiello and Srikantiah [18]. Other applications of the code include nozzle flow (Chan, Klein, and Chan [19]), reactive flow (Klein, Chan, and Chan [20]) and blast propagation (Chan and Klein [21]).

EITACC employs an Eulerian grid. A computation cell contains either all liquid, all gas, or a mixture of liquid and gas. In the problem of bubble collapse, the interface between the gas and liquid must be well defined. In the discretized space, this interface is then represented by a single layer of two-phase cells, separating the regions of single phase cells. Since the density of gas is very small compared to that of water, the dynamic effect of the gas on the liquid flow is mainly through its pressure, not its motion. We assume that the velocity is continuous across the interface following the zero normal and tangential stress condition on the interface (Harlow and Amsden [22,23]). This assumption leads to a single velocity at each mesh point and is employed rather extensively in the literature.

We neglect the motion and deformation of the plate in the simulation as such motion was observed in the experiment to be small compared to that of the bubble. In this approximation the boundary condition of the fluid is zero normal velocity on the solid surface.

In cylindrical coordinates, a computational cell is identified by the indices $i, j$, and $k$, counting in the $r-, \theta-$, and $z$-directions, respectively. We follow the staggered cell convention in discretizing the variables. Thus, the scalars, such as the partial density $m$, the material density $\rho$, and the pressure $p$ are defined in the cell center, while the velocities $u, v$, and $w$ in the $r-, \theta$-, and $z$-directions, respectively, are defined in the center of the cell faces.

Label the cell faces as im, ip, jm, jp, km, and kp, for $i-1 / 2, i+1 / 2, j-1 / 2, j+1 / 2, k-1 / 2$, and $k+1 / 2$, respectively. For the velocity $u$ at the $(n+1) t h$ time level, the finite difference momentum equation can be written as

$$
\begin{aligned}
& u_{i p}^{n+1}=u_{i p}-\Delta t\left[u_{i p}\left(\begin{array}{l}
\partial u \\
\partial r
\end{array}\right)_{i p}+{ }_{r_{i p}}^{v_{i p}}\left(\begin{array}{c}
\partial u \\
\partial \theta
\end{array}\right)_{i p}+w_{i p}\left(\begin{array}{l}
\partial u \\
\partial z
\end{array}\right)_{i p}-{ }_{i p}^{r_{i p}^{2}}\right] \\
& +\begin{array}{cc}
\Delta t & p_{i+1}^{n+1}-p^{n+1} \\
\Delta r_{i p}\left(m_{L}\right)_{i p}+(m G)_{i p}
\end{array}-\Delta t(g r)_{i p}
\end{aligned}
$$


at the ip face. Here, $\Delta t$ is the time step, $g$ is the gravitational constant, and the effects of viscosity and surface tension are assumed to be negligible. The cell index $(\mathrm{i}, \mathrm{j}, \mathrm{k})$ and the superscript for the nth time level are understood when they are not explicitly specified. The mass equation can be written as

$$
m_{\lambda}^{n+1}=m \lambda-\underset{r \Delta r \Delta \theta \Delta z}{\Delta t} F_{\lambda}
$$

where $\lambda=L$ or $G$, for liquid and gas, respectively. The mass flux $F$ is defined as

$$
\mathbf{F}=\mathbf{M}_{i p} u_{i p}^{n+1}-\mathbf{M}_{i m} u_{i m}^{n+1}+\mathbf{M}_{j p} v_{j p}^{n+1}-\mathbf{M}_{j m} v_{j m}^{n+1}+\mathbf{M}_{k p} w_{k p}^{n+1}-\mathbf{M}_{k m} w_{k m}^{n+1},
$$

where $\mathbf{M}_{i p}$, etc., are the masses of fluid being fluxed across the cell faces. The equations of state are assumed to be linear in pressure for the liquid and adiabatic and ideal for the gas.

The implicit pressure equation is obtained by first eliminating the velocities at the $(n+1) t h$ level in the mass Eq. (2) with the help of the velocity Eq. (1) and similar equations for the other velocity components. Then, eliminate the densities and partial densities at the $(n+1)$ th level using the equations of state and the equation for volume conservation,

$$
m_{G}+\begin{aligned}
& m_{L} \\
& \rho_{L}
\end{aligned}=1 .
$$

Retaining only the first order in pressure in the $(n+1)$ th time level, we can then obtain a linear equation for the pressure at the cell $(\mathrm{i}, \mathrm{j}, \mathrm{k})$ and its neighboring cells as the only unknown variables. The matrix equation obtained by collecting the pressure equations for all of the cells can be solved by the method of alternating direction line successive overrelaxation or other standard methods (see e.g., Varga [24] and Ames [25]). Once the pressure is known, all the other variables can be obtained by back substitutions.

Some quantities such as, $m_{i p}, M_{i p},(\partial u / \partial r)_{i p},(\partial u / \partial \theta)_{i p},(\partial u / \partial z)_{i p}$, etc., are to be evaluated at the cell faces and need special considerations. For the advection terms, as in (1), we need to calculate the derivatives of the velocities at the center of a cell face, with respect to the $r_{-}, \theta$-, and $z$-directions. In finite difference, this requires taking the difference of the velocities at two or more neighboring cells. On the other hand, it is important to advect the liquid with a liquid velocity, and the gas with a gas velocity. Calculation of the derivatives across the interface should be avoided. This is especially important in the later stage of the bubble collapsing process when the jet forms, since the velocity near the jet surface changes rapidly in time and space. For the interior of a single phase region, there is no problem of reaching out to a cell of different phase. We calculate the advection terms for the interior cells by the central difference, which is second order in accuracy. For the cell faces of a two-phase cell, we use a second order upwind differencing. In this second order difference scheme, we can be sure that the velocity of the advancing phase is used in calculating the advection.

The partial densities $m_{\mathbf{L}}$ and $m_{\mathbf{G}}$ in Eq. (1) are for the fluid between the centers of the neighboring cells, where the pressure difference is evaluated. For the ip face, for example, the partial densities are obtained from the liquid and gas masses $\mathbf{M}_{\mathbf{L}}$ and $\mathbf{M}_{\mathbf{G}}$ in the control volume bounded by the $i$ and $i+1$ faces, the $j m$ and $j p$ faces, and the $k m$ and $k p$ faces as shown in Fig. 1. Then, $m_{\mathbf{L}}=\mathbf{M}_{\mathbf{L}} / \mathbf{V}$ and $m_{\mathbf{G}}=\mathbf{M}_{\mathbf{G}} / \mathbf{V}$, where $\mathbf{V}$ is the volume of the control volume.

The masses $\mathbf{M}$ in Eq. (3) are calculated as follows. For the ip face, construct a control volume bounded by the streamlines and a plane at $r_{i p}-u_{i p} \Delta t$, as shown in Fig. 2. The liquid mass and the gas mass are then calculated in this control volume to obtain $\left(\mathbf{M}_{\mathbf{L}}\right)_{i p}$ and $\left(\mathbf{M}_{G}\right)_{i p}$. This construction guarantees that the correct amount of mass is transferred across the cells in pure advection.

In Eq. (3), the masses in the flux term are approximated by their values at time level $n$. This usually leads to diffusions of the interface, i.e., there may be more than one layer of two-phase cells separating the two single phase regions. In order to restore the density field to the sharp representation in our discrete space, a sharpening algorithm is applied at the end of each time step. This algorithm involves the transferring of liquid in the direction opposite to the normal of the interface, which points from liquid to gas, as follows.

Suppose that a two-phase cell $(i, j, k)$ with volume $\nu$ has an interface normal $\left(n_{r}, n_{\theta}, n_{z}\right)$ with all of the components greater than zero. Then, liquid is drawn from the cells, $(i+1, j, k),(i, j+1, k)$ and $(i, j, k+1)$ in proportion to $n_{r}, n_{\theta}$ and $n_{z}$, and deposited to the cell $(i, j, k)$. Observe that we cannot overdraw the cell's current holding of liquid, $m_{\mathbf{L}} \nu$, or overfill its available space, $\left(1-m_{\mathbf{L}} / \rho_{\mathbf{L}}\right) \nu$. We obtain the increase of the partial liquid 


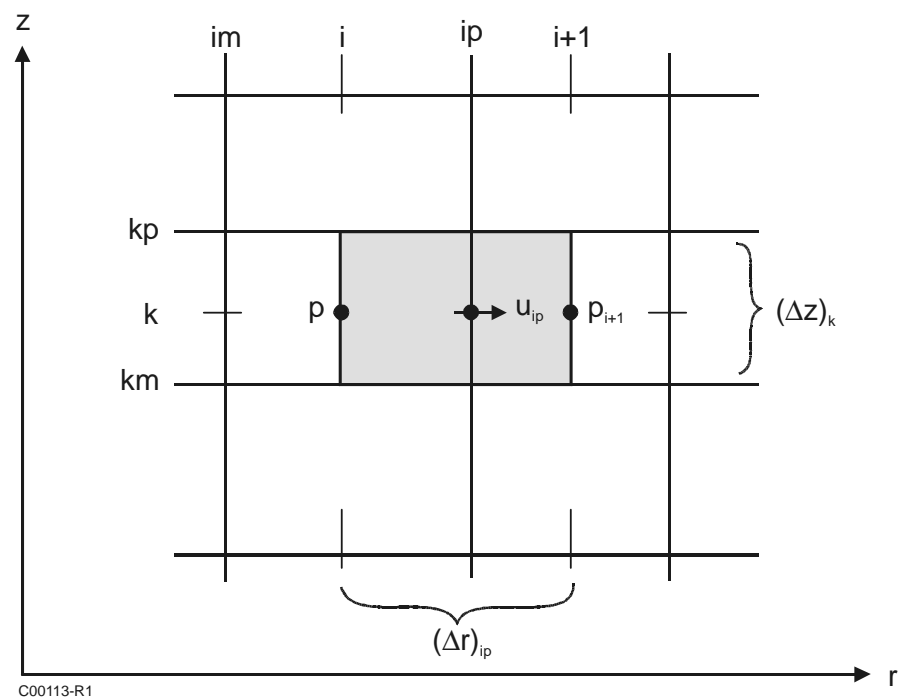

Fig. 1. Control volume for the calculation of partial densities across the ip face.

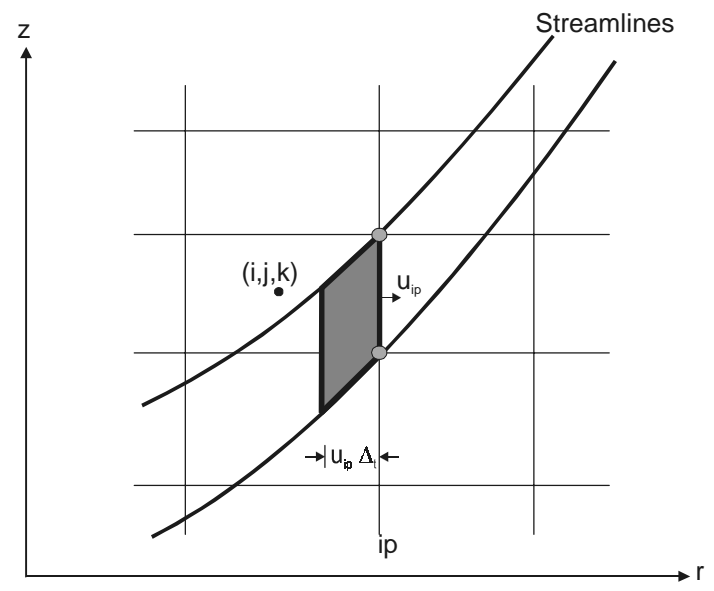

Fig. 2. Control volume for the calculation of mass flux across the ip face.

density in cell $(\mathrm{i}, \mathrm{j}, \mathrm{k})$ as

$$
\Delta m=\min \left\{\rho-m, m_{i+1} \mu / n_{r}, m_{j+1} \mu / n_{\theta}, m_{k+1} \mu / n_{z}\right\},
$$

where

$$
\mu \equiv\left(\nu_{i+1} n_{r}+\nu_{j+1} n_{\theta}+\nu_{k+1} n_{z}\right) / \nu,
$$

and the subscript $\mathbf{L}$ is suppressed for brevity. The change in the partial liquid densities in cells $(i+1, j, k)$ is given by

$$
\Delta m_{i+1}=-n_{r} \Delta m / \mu,
$$

and by similar equations for the other cells. This liquid transfer process is applied to the entire field repeatedly until we obtain only one layer of two-phase cells separating the single-phase regions.

We make use of the axial symmetry of the test configuration and set up a cylindrical coordinate system in which the z-axes points vertically upwards and passes through the charge and the center of the plate. Only two-dimensional $(r, z)$ simulations are needed under this symmetry. A typical computation domain lies completely in water and has a 


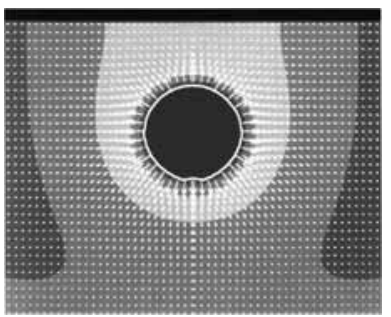

(a) Initial bubble expansion, $\mathrm{t}=0.01 \mathrm{~T}$

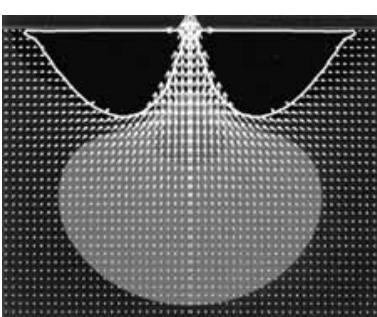

(d) Jet impact, $\mathrm{t}=1.07 \mathrm{~T}$

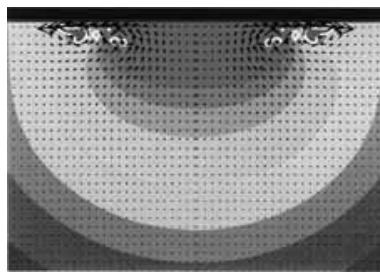

(g) Second relief flow, $\mathrm{t}=1.17 \mathrm{~T}$

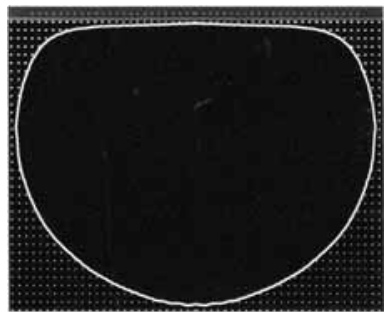

(b) Bubble maximum, $\mathrm{t}=0.63 \mathrm{~T}$

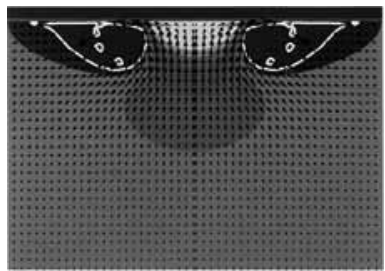

(e) Hydraulic jump and splash, $\mathrm{t}=1.14 \mathrm{~T}$

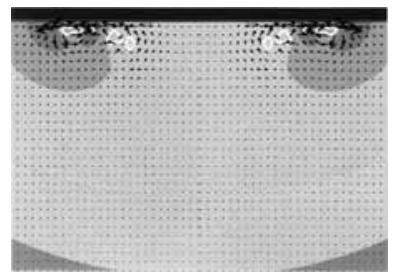

(h) Second flow impact, $\mathrm{t}=1.19 \mathrm{~T}$

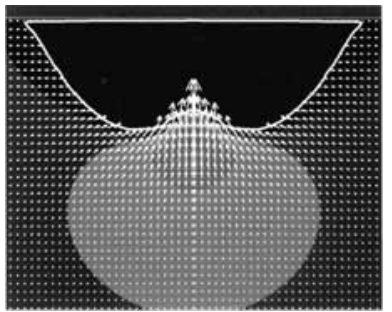

(c) Jet formation, $\mathrm{t}=1.03 \mathrm{~T}$

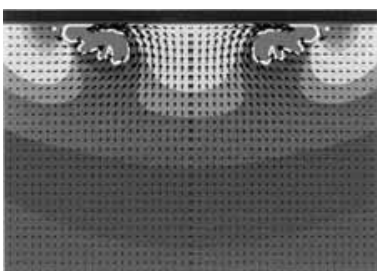

(f) Hydraulic jump impact, $\mathrm{t}=1.16 \mathrm{~T}$

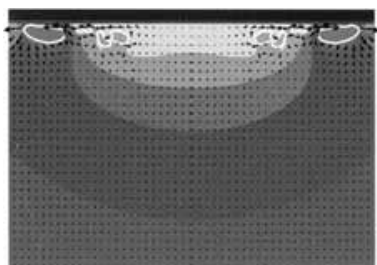

(i) Bubble re-expansion, $\mathrm{t}=1.20 \mathrm{~T}$

Fig. 3. Bubble shape, fluid velocity and pressure contours in an EITACC simulation.

dimension greater than 50 times the maximum bubble radius in all directions. The cells have uniform $\Delta_{r}$ and $\Delta_{z}$ in the central region under the plate to cover the bubble at its maximum and have gradually increasing size towards the boundaries of the computation domain. In a nominal run, we have about a hundred uniform cells in each direction in the central region.

We use pressure boundary condition at the top of the domain and solid boundary condition on the other boundaries. However, due to the large dimension of the domain, the solid boundary condition can also be replaced with the hydrostatic pressure boundary condition with negligible effect on the outcome.

The initial condition of the calculation is a spherical bubble at the Rayleigh minimum. The radius and pressure of the bubble at this minimum can be found from the weight and depth of the explosive by empirical formulas (Snay and Christian [2]). The initial fluid velocity is assumed to be zero everywhere in the computational domain.

\section{Bubble collapse phenomenon}

When an explosion is set off under a submersed plate, the plate hinders the flow on top of the explosion bubble, leading to the development of a jet in the collapsing phase of the bubble. The vertical jet will then impinge on the plate, giving rise to a concentrated loading. In Fig. 3, we show a series of pressure contour graphs from the simulation of the atmospheric pressure case. With low and high pressures represented by blue and red, respectively, and the bubble boundary shown in white curves, Fig. 3 serves as an illustration of the various stages of the collapsing process. From the study of the data and all of the simulations performed, we have arrived at a qualitative understanding of the bubble collapse phenomenon, which can be summarized in a schematic diagram (Fig. 4) for the pressure loading on the plate.

The pressure loading on the center of the plate is a result of arresting the momentum of the collapsing water jet. If the jet were infinitely wide with a flat leading edge, the loading would be controlled by the compressibility of the 


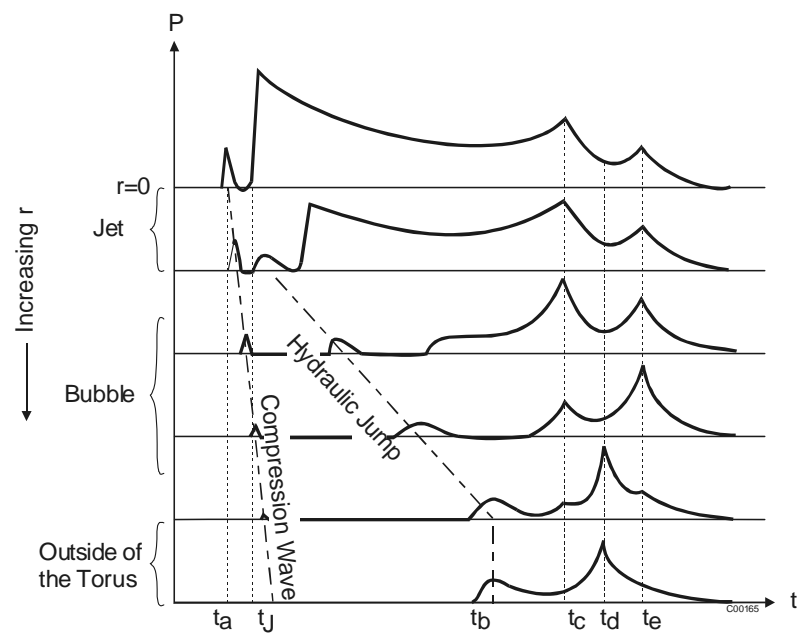

Fig. 4. Schematics of bubble loading on the plate. Times shown are: $t_{a}$, arrival of the jet at water layer; $t_{J}$, arrival of jet at solid surface; $t_{b}$, impact of hydraulic jump on outer edge of bubble; $t_{c}$, start of jet flow relief from the collapse of bubble fragments; $t_{d}$, impact of jet flow on outer edge of bubble; $t_{e}$, the collective minimum volume of the bubble fragments and the beginning of bubble re-expansion.

water, leading to a peak pressure of $\rho c v$. Because the jet is of finite width and conical shape, the jet flow is deflected to the side along the plate, and the pressure depends on the amount of relief provided by that flow. The presence of the gas bubble provides a geometric constraint that significantly affects the deflected flow.

The central pressure is relieved by three distinct processes that allow liquid flow away from the jet impact point. These three processes are (1) the radial splash along the plate while the bubble is large $\left(t_{J}<t<t_{b}\right)$; (2) the flow into the collapsing toroidal gas bubble and its fragments $\left(t_{c}<t<t_{d}\right)$; and (3) the outward flow of the entire field as the gas bubble re-expands $\left(t>t_{e}\right)$. When these processes can proceed unimpeded, the pressure drops steadily towards the ambient conditions.

The first two processes are interrupted before the pressure is completely relieved: (1) the radial splash ends at tb when the hydraulic jump impacts on the outer surface of the bubble and (2) the inward flow into the bubble ends at td as the internal pressure of the bubble rises. These events are signaled by high pressures outside the bubble and a gradual rise of pressure at the center of the plate.

Apart from the processes mentioned above, we note the collision of the jet with the water layer that lies between the plate and the bubble at $t=t_{a}$ in Fig. 4. This collision will create a compression wave that propagates through the water layer with the speed of sound. If the jet velocity is large enough, this compression wave will be recorded as a sharp and narrow peak in the beginning of the jet signal.

Note also the propagation of a pressure signal denoted as the hydraulic jump in Fig. 4. This results from the radial flow (pressure relieve process 1) on the plate surface as the flow pushes away the thin layer of stationary water originally lying between the bubble and the plate (also see Fig. 3(e)). The pressure due to this hydraulic jump is usually quite small compared to the main jet pulse and can be easily dismissed as noise in the data. However, their presence is very consistent in all of the test data. The present study by computer simulation has revealed the underlying mechanism.

The collapse of the bubble fragments and the collisions of the radial flow with the water outside the toroidal bubble can both lead to high pressure on the plate in the vicinity of the minimum torus (Fig. 3(f-i)). The work of Tomita and Shima [6] has shown that the cavitation damage is concentrated at this ring-shaped site. The double peak nature of the loading near the torus minimum observed here should be studied in connection of the damage mechanism.

\section{Comparison with data}

In Fig. 5, the calculated pressure-time histories for the normal atmospheric pressure case are compared with the experimental pressure records at stations $R=n D R, n=0,1,2, \ldots$, with equal spacing $D R \approx 1 / 12 A_{\max }$ from 


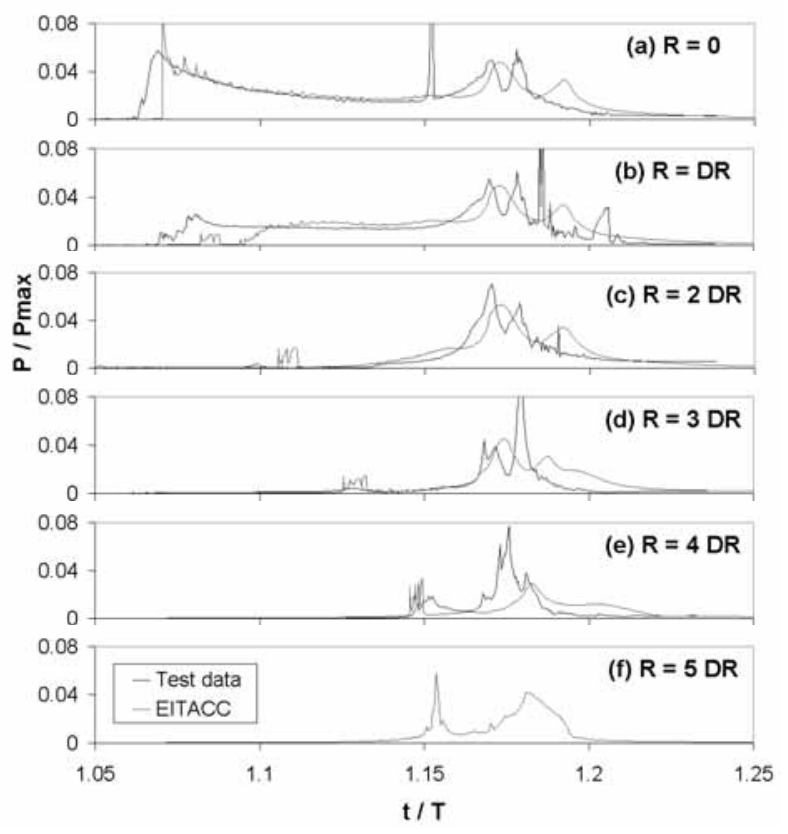

Fig. 5. Comparison of the EITACC predicted pressure loading (red curves) with data (blue curves).

the center of the plate. The maximum radius of the Rayleigh bubble, $A_{\max }$, is approximately equal to the maximum radius of the bubble shown in Fig. 3(b), as the bubble there is not too far from the fully expanded shape. The pressure and time in Fig. 5 are normalized by the Rayleigh period $T$ and the maximum Rayleigh pressure $P_{\max }$. The Rayleigh parameters $A_{\max }, T$, and $P_{\max }$ are related to the charge weight and the explosion conditions and the relationships can be found in Snay and Christian [2].

We should point out that the pressure data shown in Fig. 5 contains some noise in the form of sharp spikes as in $1.15 \mathrm{~T}<t<1.16 \mathrm{~T}$ for $R=0$ and $1.18 \mathrm{~T}<t<1.19 \mathrm{~T}$ for $R=D R$. These spikes are noise as they are not repeatable in the test. Discounting the noise, we can see in Fig. 5 that the calculated pressure agrees with the data very well.

The time history at $R=0$ (Fig. 5(a)) shows an overall agreement with the data. Although the calculation overshoots the jet impact peak in one time step, the calculated pulse matches the exponential decay and the duration of the data very well. Considering the time histories at different locations simultaneously (Fig. 5), we see the qualitative features of the pressure relief processes discussed previously (Fig. 4). In particular, one can clearly identify the signals from the hydraulic jump and from the position of these signals at different $\mathrm{R}$, this hydraulic jump propagates with a velocity in good agreement with the data (Fig. 5).

The double peak structure at the end of the pulse $(1.15 \mathrm{~T}<t<1.20 \mathrm{~T})$ for the traces at $R \leqslant 3 D R$ and the single peak structure there for the trace at $R=4 D R$ are also reproduced by the calculation (Fig. 5). These peaks are due to the breaking up of the torus and are somewhat wider in the simulation as compared to those of the data.

The pressure trace at $R=\Delta R$ in Fig. 5 shows that the calculated jet pulse arrives somewhat later as compared to the data. This implies that the jet is slightly sharper in the calculation than it is in the test.

The sensitivity of spatial resolution in the simulation is demonstrated in Fig. 6 where the pressure at $R=0$ for three grid choices are compared. It shows that the results with different grids are very consistent, with improved agreements with the data for finer grids, as expected.

Results for the reduced pressure case can be found in Kan and Stuhmiller [26].

\section{Discussions}

The treatment of the advection term has a strong effect on the formation of the jet. In Chan's study [16] where the first order upwind differencing was used, the jet formation was much delayed, resulting in a pulse duration of about 


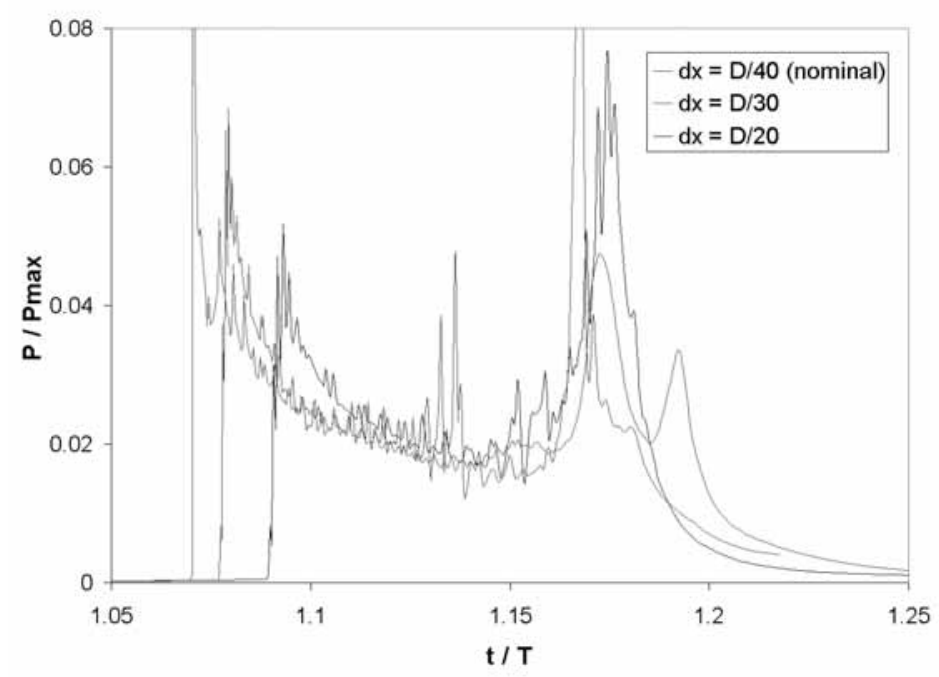

Fig. 6. Comparison of the pressure loading on the plate at $R=0$ from three EITACC simulations with different spatial resolutions.

half of the experimental value. When central differencing was used the duration of the pulse immediately increased to about three quarters of the experimental value. However, the bubble surface became rough, due to the mixing of the liquid and gas velocities across the interface. The achievement of good pulse duration and a smooth bubble surface is secured only when the present hybrid central-upwind advection scheme is employed.

For the calculation of the mass in the flux in Eq. (3), Chan [16] used the density of the upwind cell. This scheme creates unbalanced mass at the interface even for a pure translation parallel to the grid lines. It did not lead to a problem in combination with his treatment of the advection term probably due to the highly diffusive nature of the upwind differencing. However, when the more accurate advection scheme was employed, the unbalance mass generated persistent negative pressures at the interface. This problem was under control only when the present method of calculating the masses $\mathrm{M}$ in Eq. (3) from the control volume defined by the stream lines (Fig. 2) was employed.

The pressure traces in Figs 5 and 6 show some sharp spikes especially near the impact of the jet front. This is due to the propagation of the interface across the cell boundaries. Note that the net liquid flux should be very close to zero for a full liquid cell due to the fact that liquids are highly incompressible. On the other hand, the net liquid flux into a two-phase cell is nonzero, unless the interface is stationary. There may be a situation that a two-phase cell becomes a full liquid cell after the packing of the interface. The newly filled liquid cell may have a small unbalanced liquid flux that can cause some numerical pressure oscillations. The pressure usually goes back to normal in a few time steps when such a situation no longer exits and we should let this relaxation run its course so that the incorrect pressure does not propagate to later times. Therefore, smaller time steps should be used if the relaxation is interrupted by consecutive interface crossings.

\section{Conclusion}

We have successfully applied an Eulerian, two-fluid CFD code to simulate the collapse of a bubble generated by an underwater explosion. We have shown that nearly every aspect of the observed pressure loading, including small features that might have been dismissed as spurious "noise," can be understood and reproduced, when the advection and mass flux at the interface are treated with good accuracy. 


\section{Acknowledgment}

The authors wish to thank John F. Goertner, Thomas Farley and Gregory S. Harris at the Naval Surface Warfare Center for numerous penetrating discussions throughout the course of the work. The support of this work was provided under Contract No. N60921-92-C-0048 from US Office of Naval Research.

\section{References}

[1] M. Kornfeld and L. Suvorov, On the Destructive Action of Cavitation, J. Appl. Phys. 15 (1944), $495-506$.

[2] H.G. Snay and E.A. Christian, Underwater Explosion Phenomena: The Parameters of a Non-migrating Bubble Oscillating in an Incompressible Medium, NAVORD Report 2437, 1952.

[3] C.F. Naude and A.T. Ellis, On the Mechanism of Cavitation Damage by Nonhemispherical Cavities Collapsing in Contact with a Solid Boundary, Trans. ASME D: J. Basic Engng. 83 (1961), 648-656.

[4] T.B. Benjamin and A.T. Ellis, The Collapse of Cavitation Bubbles and the Pressure Thereby Produced Against Solid Boundaries, Phil. Trans. R. Soc. A $\mathbf{2 6 0}$ (1966), 221-240.

[5] M.S. Plesset and R.B. Chapman, Collapse of an Initially Spherical Vapour Cavity in the Neighbourhood of a Solid Boundary, J. Fluid Mech. 47 (1971), 283-290.

[6] Y. Tomita and A. Shima, Mechanisms of Pressure Generation and Damage Pit Formation by Bubble Collapse, J. Fluid Mech. 169 (1986), 535-564.

[7] J.R. Blake, B.B. Taib and G. Doherty, Transient Cavities near Boundaries, Part 1, Rigid Boundary, J. Fluid Mech. 170 (1986), $479-499$.

[8] J.R. Blake and D.C. Gibson, Cavitation Bubbles Near Boundaries, Ann. Rev. Fluid Mech. 19 (1987), 99-123.

[9] J.H. Duncan and S. Zhang, On the interaction of a collapsing cavity and a compliant wall, J. Fluid Mech. 226 (1991), 401-423.

[10] Rayleigh, Load, On the Pressure Developed in a Liquid During the Collapse of a Spherical Cavity, Phil. Mag. 34 (1917), 94-98.

[11] J.F. Goertner, R. Thrun and J.E. Berry, Underwater Explosion Bubble Collapse Against a Flat Plate, 1987 NSWC Hydrotank Test Series Pressure Data Report, NSWC Report NSWCDD/TR-93/98, Limited distribution, 1993.

[12] J.R. Blake and A. Prosperetti, Dynamics of Underwater Explosion Bubbles, Final Report to the Office of Naval Research, Grant No. N0001489-J-1791, May 1989.

[13] G.L. Chahine, A Numerical Model for Three-Dimensional Bubble Dynamics in Complex Flow Configurations, A paper presented at the 22nd ATIC meeting, St. John's, Newfoundland, Canada, Aug. 1989.

[14] G.L. Chahine, Bubble/Bubble and Bubble/Flow Strong Interactions, presented at the IUTAM Symposium on Bubble Dynamics and Interface Phenomena, Sept. 4-10, 1993.

[15] J.P. Best, The formation of toroidal bubbles upon the collapse of transient cavities, J. Fluid Mech. 251 (1993), $79-107$.

[16] R.K.-C. Chan, Validation of The EITACC-FS Computer Code for Underwater Explosion Analysis, JAYCOR Final Report for Naval Surface Weapon Center, White Oak, Contract No. N60921-86-C-0148, 1988.

[17] D.R. Liles and W.H. Reed, A Semi-Implicit Method for Two-Phase Fluid Dynamics, J. Comp. Phys. 26 (1978), $390-407$.

[18] R.K.-C. Chan, P.J. Masiello and G.S. Srikantiah, PORTHOS - A Computer Code for Solving General Three-Dimensional, Time-Dependent Two Fluid Equations, ASME Paper 86-WA/NE-3 presented at the Winter Annual Meeting, Anaheim, Ca., Dec. 1986.

[19] P.C. Chan, H.H. Klein and R.K.-C. Chan, Computational Analyses of Subscale Space Shuttle Main Engine Nozzles at 3.2-sec Shutdown Condition, AIAA paper 88-3200, presented at the 24th Joint Propulsion Conference and Exhibit, Boston, MA, July 11-13, 1988.

[20] H.H. Klein, P.C. Chan and R.K.-C. Chan, JAYCOR CFD Analysis of the Hydrogen Disposal System at the Vandenberg Space Shuttle Lunch Site, AIAA paper 89-0579, presented at the 27th Aerospace Sciences Meeting, Reno, NV, Jan. 9-12, 1989.

[21] P.C. Chan and H.H. Klein, A Study of Blast Effects Inside an Enclosure, J. Fluids Eng. 116 (1994), $450-455$.

[22] F.H. Harlow and A.A. Amsden, A Numerical Fluid Dynamics Calculation Method for All Flow Speeds, J. Comp. Phys. 16 (1971), 1-19.

[23] F.H. Harlow and A.A. Amsden, Multifluid Flow Calculations at All Mach Numbers, J. Comp. Phys. 8 (1974), $197-213$.

[24] R.S. Varga, Matrix Iterative Analysis, Prentice-Hall, Englewood Cliffs, NJ, Chapter 6, 1962.

[25] W.F. Ames, Numerical Methods for Differential Equations, 2nd Edition, Academic Press, New York and London, Chapter 3, 1977.

[26] K.-K. Kan and J.H. Stuhmiller, The Phenomena of Underwater Explosion Bubbles Under a Circular Plate, NSWC Final Report, Contract No. N60921-92-C-0048, Apr. 20, 1994, Limited distribution. 

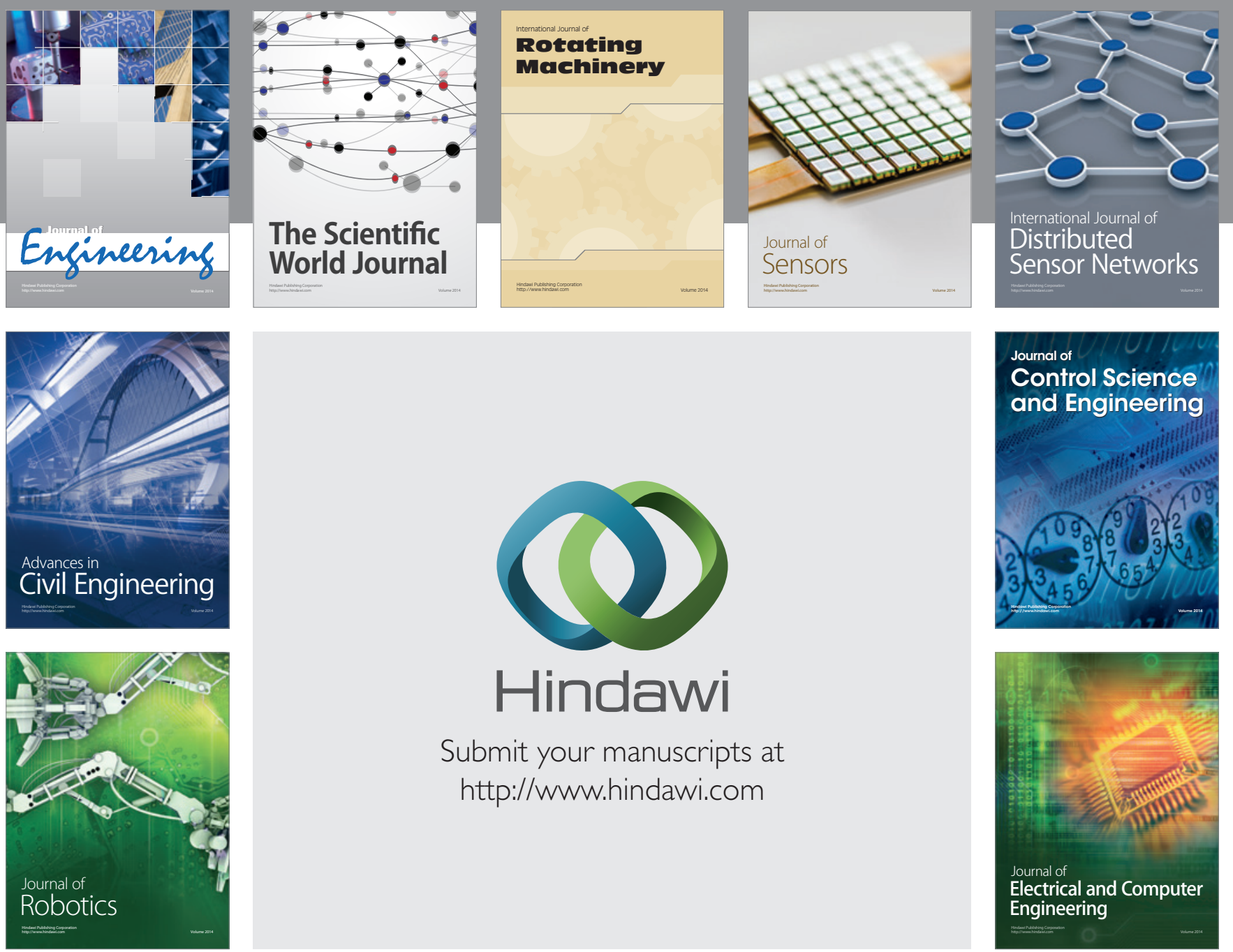

Submit your manuscripts at

http://www.hindawi.com
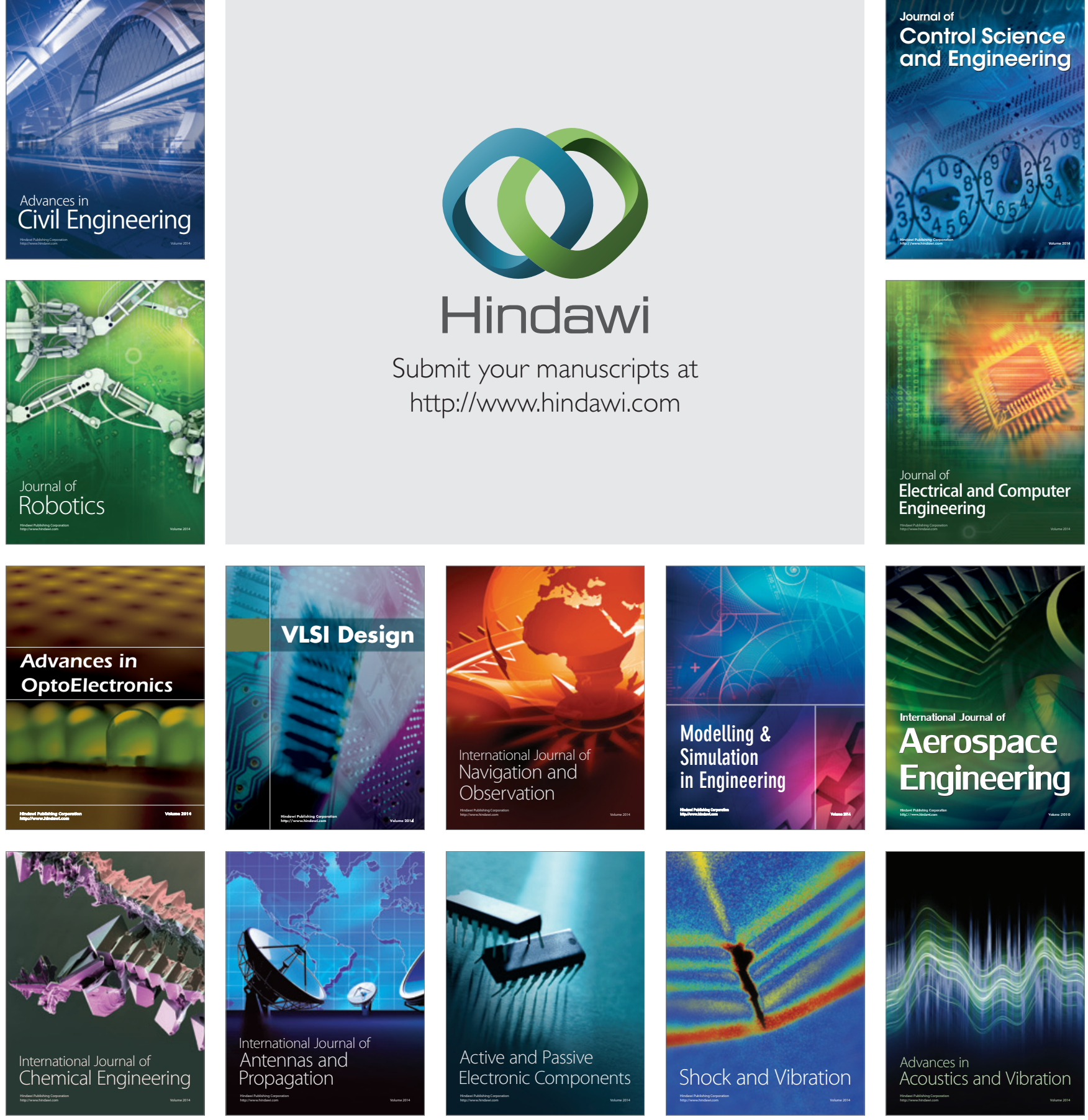